\title{
Towards Automated EEG-Based Alzheimer's Disease Diagnosis Using Relevance Vector Machines
}

\author{
Raymundo Cassani*, Tiago H. Falk*, Francisco J. Fraga ${ }^{\dagger}$, Paulo A. Kanda ${ }^{\ddagger}$, Renato Anghinah ${ }^{\ddagger}$ \\ ${ }^{*}$ Institut National de la Recherche Scientifique (INRS-EMT), University of Quebec, Canada \\ ${ }^{\dagger}$ Engineering, Modelling and Applied Social Sciences Center, Universidade Federal do ABC, Brazil \\ ${ }^{\ddagger}$ Reference Center of Behavioural Disturbances and Dementia, Universidade de São Paulo, Brazil
}

\begin{abstract}
Existing electroencephalography (EEG) based Alzheimer's disease (AD) diagnostic systems typically rely on experts to visually inspect and segment the collected signals into artefact-free epochs and on support vector machine (SVM) based classifiers. The manual selection process, however, introduces biases and errors into the diagnostic procedure, renders it "semiautomated," and makes the procedure costly and labour-intensive. In this paper, we overcome these limitations by proposing the use of an automated artefact removal (AAR) algorithm to remove artefacts from the EEG signal without the need for human intervention. We investigate the effects of the so-called waveletenhanced independent component analysis (wICA) AAR on three classes of EEG features, namely spectral power, coherence, and amplitude modulation, and ultimately, on diagnostic accuracy, specificity and sensitivity. Furthermore, we propose to replace the binary SVM classifier with a soft-decision relevance vector machine (RVM) classifier. Experimental results show the proposed RVM-based system outperforming the SVM trained on features extracted from both manually-selected and wICAprocessed epochs. Moreover, the class membership information output by the RVM is shown to provide clinicians with a richer pool of information to assist with AD assessment.
\end{abstract}

Keywords-Alzheimer's disease, electroencephalography, support vector machine (SVM), relevance vector machine (RVM), wICA.

\section{INTRODUCTION}

Alzheimer's disease (AD) is an irreversible chronic neurodegenerative condition ranked as the sixth leading cause of death in the United States. Moreover, AD is the most common cause of dementia, accounting for $60-70 \%$ of dementia cases around the world. The term 'dementia' is used to encompass a number of neural conditions that cause a decline and eventually loss of cognitive functions (e.g. memory, reasoning, communication) and behavioural functions that interfere with the individual's daily life. In 2013, 5 million people were diagnosed with $\mathrm{AD}$ in the United States alone, and this number is projected to grow to 13 million by 2050 . This translates to a monetary cost (associated with $\mathrm{AD}$ and other types of dementia) of $\$ 203$ billion; by 2050 , this is forecast to reach $\$ 1.2$ trillion per year. Given the projected prevalence and economic cost of $\mathrm{AD}$, governments and the global health community are elaborating coordinated plans to take actions against the disease [1], [2]. In its 2012 dementia report, the World Health Organization placed special emphasis on early diagnostics, thus allowing for treatment to start early [3].

Today, $\mathrm{AD}$ diagnosis is commonly made by taking into account medical history, mental status examinations, laboratory tests and, recently, brain imaging tools such as computed tomography $(\mathrm{CT})$, and functional magnetic resonance imaging (fMRI). These clinical assessment methods, however, require experienced clinicians and exhaustive testing sessions. Furthermore, access to such neuroimaging tools is costly and scant, even in developed countries (e.g., in Canada, the wait time for a non-emergency fMRI can be as high as six months). These constraints are further heightened in medium- and lowincome countries, thus hindering the implementation of very early disease diagnosis worldwide [4].

To overcome these abovementioned limitations, quantitative electroencephalography (qEEG, henceforth referred to as "EEG") has been proposed as a promising tool to assist physicians in the diagnosis of AD [5]-[7]. The EEG signal is a record of the change of the electrical field produced by neural activity on the cerebral cortex. As such, EEG can be use to evaluate neuronal degeneration and functional impairment long before actual tissue loss can be detected by fMRI [1]. Several different effects of AD on the EEG signal have been reported in literature. The most distinctive of these effects include: i) noticeable shift occurring in the EEG power spectrum (e.g., slowing of the EEG) [8]-[10]; ii) reduced inter-hemispherical spectral coherence [11]-[13]; iii) decreased EEG "complexity," likely caused by the reduction in nonlinear connections between cortical regions or even neuronal death [7]; and more recently, iv) a change in neuromodulatory behaviour measured via EEG amplitude modulation analysis [5], [14]. Many of these changes have been shown to be related [15] and diagnostic accuracies in line with more advanced neuroimaging techniques (e.g., fMRI) have been achieved [16].

Notwithstanding, despite the fact that EEG recording devices offer several cost, portability, and ergonomic advantages over e.g., fMRI, EEG signals are inherently prone to several artefacts and noise sources, such as eye blinks, power grid interference, and hardware-inherent noise, to name a few. Since artefacts can have detrimental effects on EEG-based $\mathrm{AD}$ diagnostics, the majority of the published works have utilized artefact-free EEG segments (called epochs) which have been selected by expert clinicians through exhaustive visual inspection. This manual selection process introduces additional biases and errors into the diagnostic procedure [17], as well as renders it "semi-automated" [5], thus still making it costly and time-consuming. As an alternative, automated artefact removal (AAR) algorithms may be used to remove artefacts from the EEG signal without the need for human intervention.

Several AAR algorithms have been proposed in the literature, such as wavelet denoising [18], blind source separation [19], [20], and even simple feature averaging [14]. This paper 


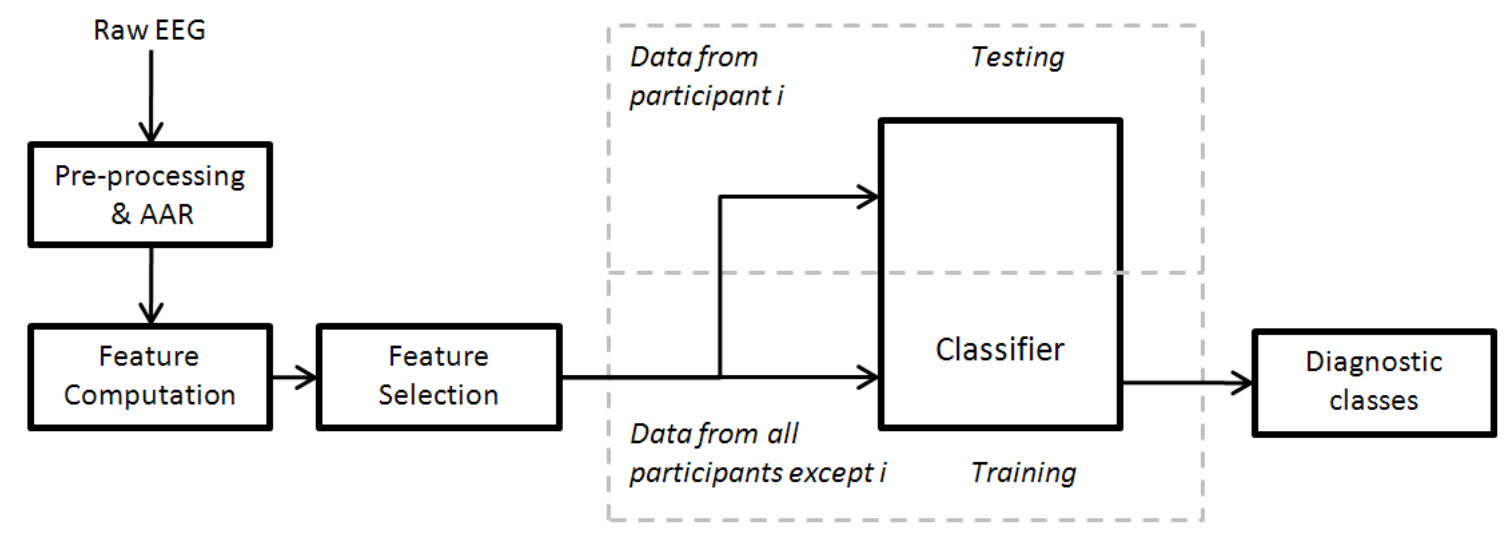

Fig. 1. Block diagram of an automated EEG-based AD diagnosis system, as well as its use in a leave-one-subject-out cross validation task.

investigates the benefits of using AAR over manual selection for automated assessment, with the end goal of developing a tool that can be used to aid clinicians (both experienced and inexperienced) during the AD diagnostic procedure. Building on recent evidence that support vector machine (SVM) based classifiers are useful for semi-automated AD diagnostics [5], [21], [22], a secondary aim of this paper is to explore the benefits of using so-called relevance vector machines (RVM) over SVM classifiers. RVMs utilize a probabilistic Bayesian learning framework to find classifiers that have i) fewer basis functions than a comparable SVM and ii) the benefit of explicitly predicting the probability of class membership, thus providing clinicians with a richer pool of information.

The remainder of this paper is organized as follows. Section II describes the methods and materials used in the present study. Section III presents the experimental results and a brief discussion. Lastly, Section IV presents the conclusions.

\section{Methods AND MATERIALS}

This section presents the details about the methods used in this study, as well as a description of the signal processing steps involved in the proposed system depicted by Figure 1 .

\section{A. Participants}

Data used in this study was obtained from fifty-nine participants who were recruited from the Behavioral and Cognitive Neurology Unit of the Department of Neurology and the Reference Center for Cognitive Disorders at the Hospital das Clinicas in São Paulo, Brazil [23]. AD patients were diagnosed by experienced neurologists according to NINCDS-ADRDA criteria [24] and classified based on the Brazilian version of the Mini-Mental State Examination (MMSE) [25]. Participants were divided in two groups. The first group $(H)$ consisted of 24 cognitively healthy controls ( 12 males; mean age 66.3 years, $8.8 \mathrm{sd}$ ), whereas the second group $(A D)$ comprised 35 mild-to-severe $\mathrm{AD}$ patients (15 males, mean age 74.8 years, $8.9 \mathrm{sd})$. Inclusion criteria for the $H$ group included a CDR score $=0$ and MMSE score $\geq 25$ (mean 28.5, $1.7 \mathrm{sd}$ ), as well as no indication of functional cognitive decline. Inclusion criteria for the $A D$ group, in turn, included $0.5 \leq \mathrm{CDR}$ $\leq 2$ and MMSE $\leq 24$ (mean 16.4, 6.0 sd). An additional criterion for inclusion to the $\mathrm{AD}$ group was the presence of functional and cognitive decline over the previous 12 months based on detailed interviews with knowledgeable informants. Additionally, the members of the AD group were screened for diabetes mellitus, kidney disease, thyroid disease, alcoholism, liver disease, lung disease or vitamin B12 deficiency, as these conditions can also cause cognitive decline. Ethics approval was obtained from the Research Ethics Office and participants consented to participate in the study.

\section{B. EEG acquisition, pre-processing, and AAR}

Twenty-channel EEG signals were acquired with the participants awake, relaxed, and with their eyes closed for at least eight minutes. EEG was recorded with 12-bit resolution and $200 \mathrm{~Hz}$ sampling frequency using Braintech 3.0 instrumentation (EMSA Equipamentos Médicos INC., Brazil). Scalp electrodes were placed according to the International 10-20 montage system, as depicted by Fig. 2, electrode impedance was kept below $10 \mathrm{k} \Omega$, and bi-auricular (attached) electrodes were used as reference. An infinite impulse response low-pass elliptic filter with a zero at $60 \mathrm{~Hz}$ was applied to eliminate power grid interference. In addition to the EEG channels recorded from the scalp electrodes, eight bipolar signals, which are mathematically computed as the subtraction of the two bi-auricular signals involved, were computed[26]. The eight bipolar signals used were the inter-hemispheric signals Fp1-Fp2, F7-F8, F3F4, T3-T4, C3-C4, T5-T6, P3-P4, and O1-O2.

Unprocessed raw EEG signals underwent visual inspection by two experienced clinicians to remove intervals contaminated by eye blinking, muscle movements, drowsiness, hardwareinherent noise, or any other sort of artefact. These manuallyselected clean EEG signals were then separated into 8-second epochs, which were used in this study to train a baseline "semi-automated" classifier to benchmark the AD diagnostic performance obtained with the proposed fully-automated systems. For the proposed system (see Fig. 1), the raw EEG is further processed by a state-of-the-art AAR algorithm. Motived 


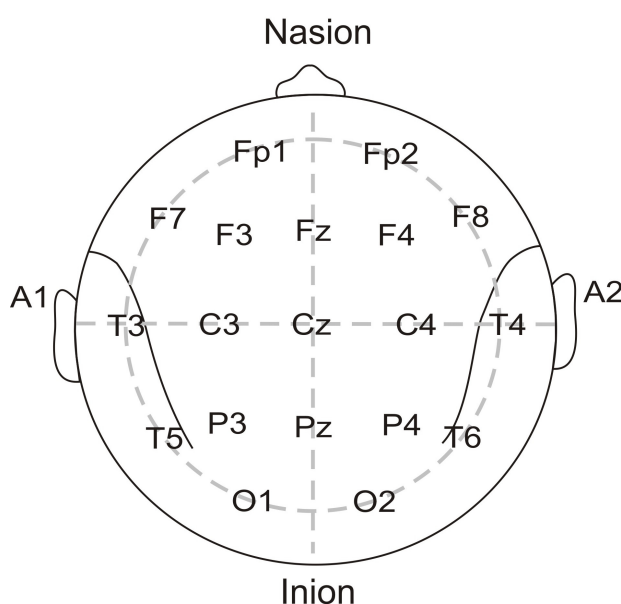

Fig. 2. EEG electrode positioning following the International 10-20 System

by our recent findings [27], we used the wavelet-enhanced independent component analysis (wICA) algorithm [28].

The wICA algorithm applies a thresholding process based on wavelets to the demixed independent components with the finality of recovering any residual neural activity that may be present in components marked as artefactual. The wICA method is summarized in five steps: (1) the EEG data is decomposed into independent components (IC); (2) the wavelet transform is applied to the ICs; (3) thresholding of the wavelet coefficients is performed to differentiate between neural and artefactual coefficients; (4) the inverse wavelet transform is applied to the thresholded coefficients, retrieving ICs with only neural activity; and lastly, (5) wavelet-corrected ICs are projected to obtain the artefact-free EEG data. The wICA algorithm provides an improved performance and better preservation of EEG spectral and phase coherence properties compared with ICA algorithm [28], [29]. For illustration purposes, Figure 3 depicts a representative EEG segment before (blue) and after wICA processing (red). To allow for direct comparison with the benchmark system, the EEG signal processed with wICA was divided into 8s epochs.

\section{Feature Computation}

The next stage in $\mathrm{AD}$ diagnosis comprises feature computation. Here, we have used previously-proposed features shown to reliably characterize $\mathrm{AD}$; features can be grouped into three classes: spectral, coherence, and amplitude modulation. Spectral power features measure the power present in each of the five conventional EEG frequency bands, namely: $0.1-4$ $\mathrm{Hz}$ (delta), $4-8 \mathrm{~Hz}$ (theta), $8-12 \mathrm{~Hz}$ (alpha), $12-30 \mathrm{~Hz}$ (beta) and, $30-100+\mathrm{Hz}$ (gamma) [30]. These five spectral power features were computed per epoch for each of the 20 EEG electrodes, as well as the 8 virtual bipolar signals, thus resulting in 140 features. Coherence features, in turn, measure the co-variance between either two power spectra or two EEG phase signals (termed magnitude square coherence or phase coherence, respectively). These features have been used as indicators of cortical connectivity [31]. As we are interested inter-hemispheric connectivity, coherence features (both magnitude and phase) were computed only for the 8

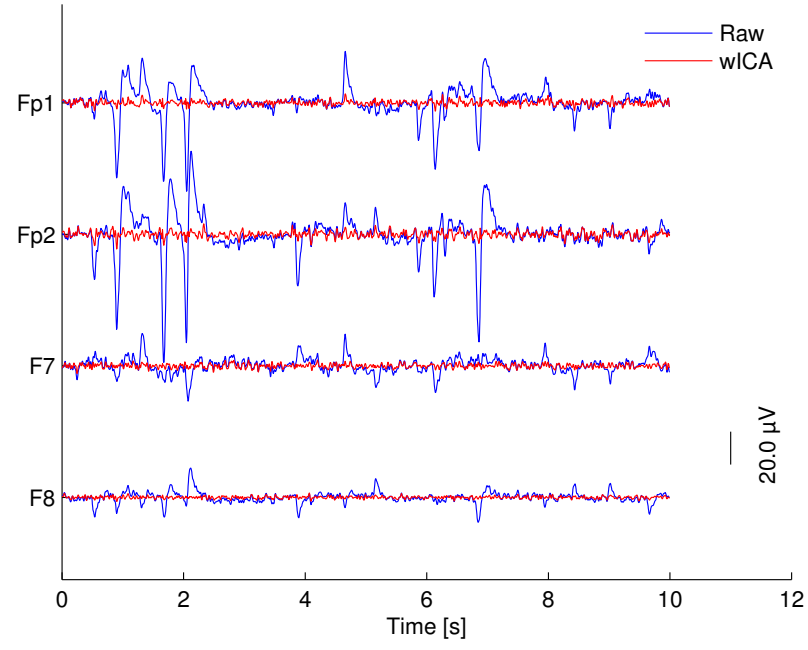

Fig. 3. Plots of raw (blue) and wICA-processed (red) EEG signals for four of the collected 20 electrodes.

bipolar signals, for each of the five frequency bands, thus resulting in 80 coherence-based features.

Lastly, amplitude modulation features have been recently proposed and shown to reliably detect $\mathrm{AD}$, as well as monitor disease progression [5], [32], [33]. To compute the features, the EEG full-band signal is first decomposed into the five conventional sub-bands: delta, theta, alpha, beta, and gamma. Next, each subband envelope is computed via a Hilbert transform. Lastly, in order to characterize the dynamics of the amplitude modulations, a second frequency decomposition is performed on the band envelope signals. To characterize the crossfrequency interactions, this second decomposition utilizes five so-called "modulation bands" that have been designed to coincide with the frequency ranges of the five traditional subbands. The normalized power in each frequency-modulation band is used as a feature. It is important to note that due to the Bedrosian's theorem [34], not all frequency-modulation band combinations make sense. Using the notation ' $E$ (frequency band; modulation band)' to denote the normalized energy in a given frequency and modulation band, only the following scenarios are relevant: $E$ (delta; $m$-delta), E(theta; $m$-delta, $m$ theta), E(alpha; $m$-delta, $m$-theta), E(beta; $m$-delta, $m$-theta, m-alpha, $m$-beta) and, E(gamma; m-delta, m-theta, m-alpha, $m$-beta, m-gamma). As such, these 14 features are computed for each of the 20 EEG signals, as well as the 8 virtual bipolar signals. A total of 612 features were computed for each 8second epoch available in the collected database.

\section{Feature Selection and Classification}

As mentioned above, a total of 612 features have been computed per EEG epoch. Building classifiers with such highdimensionality using limited data results in overfitting. As such, feature selection is required. From the available EEG data, we have set aside $25 \%$ of the data (randomly selected) to perform automated feature selection and have left the remaining $75 \%$ for classifier training and testing. Motivated by recent findings which have advocated for the use of support vector machines for feature selection and classification [5], [21], we 
TABLE I. LIST OF TOP-24 SELECTED FEATURES FROM MANUALLY-SELECTED AND WICA-PROCESSED EPOCHS.

\begin{tabular}{ccc}
\hline Ranking & Manual & wICA-AAR \\
\hline \hline 1 & O1_O2_theta_pwr & PZ_alpha_pwr \\
2 & F7_F8_alpha_cohe_pha & P3_alpha_pwr \\
3 & PZ_alpha_pwr & P3_P4_delta_cohe_mag \\
4 & FP2_beta_pwr & P3_delta_pwr \\
5 & T6_beta_m-delta & T3_delta_pwr \\
6 & O1_delta_pwr & F8_beta_pwr \\
7 & OZ_beta_pwr & C3_C4_beta_m-beta \\
8 & C4_delta_m-delta & O1_O2_theta_pwr \\
9 & PZ_delta_pwr & FZ_beta_m-alpha \\
10 & FP2_theta_m-delta & O1_O2_beta_cohe_mag \\
11 & CZ_beta_m-theta & F3_F4_theta_pwr \\
12 & C3_beta_m-delta & T3_T4_delta_pwr \\
13 & OZ_beta_m-beta & F7_delta_pwr \\
14 & FP1_beta_m-alpha & T3_T4_theta_pwr \\
15 & FP1_delta_pwr & F3_F4_beta_pwr \\
16 & O1_O2_alpha_pwr & C4_beta_pwr \\
17 & O1_beta_pwr & F3_F4_delta_cohe_mag \\
18 & CZ_beta_m-alpha & T3_T4_beta_cohe_mag \\
19 & T6_delta_m-delta & FP1_FP2_theta_pwr \\
20 & F8_beta_m-alpha & FZ_alpha_pwr \\
21 & F3_F4_delta_cohe_mag & FZ_alpha_m-delta \\
22 & F7_theta_m-theta & C3_delta_m-delta \\
23 & P3_P4_theta_cohe_mag & T3_beta_m-beta \\
24 & T4_theta_pwr & T3_beta_m-alpha \\
\hline
\end{tabular}

have used Weka's open source software implementation to rank the 612 features and we have used the top- 24 in our analysis. Table I lists the top- 24 selected features from the manually-selected artefact free epochs, as well as from the EEG epochs processed by the wICA AAR algorithm. Feature names are reported as "electrode_band_feature," where "electrode" represents either the 10-20 electrode positions (e.g., PZ) or the virtual bipolar signal (e.g., P3-P4), "band" represents the EEG frequency band (e.g., delta), and "feature" provides a descriptive indication of the feature representation (e.g., 'pwr' corresponds to spectral power; 'm-alpha' to modulation rate; 'cohe_mag/pha to magnitude/phase coherence).

For classification, we investigate the use of support vector machines (SVM) [35], as well relevance vector machines (RVM) [36]. The principle behind SVMs is to find an optimal hyperplane in some higher dimensionality that linearly separates data points belonging to two classes ("hard classification"). Relevance vector machines, in turn, make use of Bayesian estimation theory to output predictions that are expressed as probabilities of class memberships ("soft classification"). A secondary aim of this study is to explore the gains obtained with RVM over SVMs, as well as benefits of probabilistic outputs over binary decisions.

\section{E. Classification Performance Comparison}

As shown within the dashed lines in Fig. 1, the classification performance calculation used in this study was comprised of the leave-one-subject-out (LOSO) cross-validation approach computed with the disjoint $75 \%$ of the data that was set aside for training/testing. Within this methodology, data (i.e., feature vectors comprised of the top- 24 features selected using
$25 \%$ of the available data) from $\mathrm{N}-1$ subjects are used to train the SVM or RVM classifiers, which are then tested on data from the "left-out" subject. This procedure is repeated for all 59 participants in the dataset. A leave-one-subject-out cross-validation paradigm is useful as it avoids overfitting and guarantees the generality of the classifier to unseen data.

While classification is done on a per-epoch basis, results are reported on a per-patient basis. With this approach and the SVM classifier, a patient is labeled as healthy $(H)$ or $A D$ based on majority vote, i.e., if $50 \%$ or more of the epochs belong to a given class, thus placing equal weight on all classified epochs. With the RVM, on the other hand, the probabilistic outputs can be used to perform a better-informed majority vote fusion. For example, majority vote can be performed only on epochs that received a class membership threshold greater than $X \%$. Here, we explore the effects of " $X$ " on overall diagnostic accuracy and test values of $X=50-95 \%$ in 5\% increments. Performance comparisons are given as a function of diagnostic accuracy, sensitivity, and specificity.

In our experiments, the open-source Matlab Pattern Recognition Toolbox was used for SVM/RVM training. Polynomial kernels were used for both classifiers with a regularization coefficient $\mathrm{C}=1$ and $\gamma=0.01$. As mentioned previously, per-patient diagnostic accuracies of the automated systems are compared to the benchmark system trained on manuallyselected artefact-free EEG epochs and using an SVM classifier.

\section{EXPERIMENTAL RESULTS AND DISCUSSION}

Figure 4 depicts the performance metrics obtained for the RVM-based system as a function of the class membership threshold $X$. As can be seen, diagnostic accuracy, sensitivity and specificity is collectively optimized for a threshold between $X=70-75 \%$, after which point the performances monotonically decrease. As such, we use the $75 \%$ threshold in the proposed system. To get a sense of the distribution of EEG epochs that achieved such high class membership probability, the plot in Figure 5 can be used. The plot shows which of the over 300 epochs, per participant, were above (non-shaded) or below (shaded) $75 \%$ class probability. As can be seen, the majority of the epochs were classified with high reliability. Representative participants that had the majority of their epochs classified with $75 \%$ probability include participants 2 , 3 , and 45 , to name a few. On the other hand, participants such as number 20 and 58 had the majority of their epochs classified with less than $75 \%$ class membership probability. As can be seen, the RVM classifier provides a richer pool of data for clinicians to use in their assessment relative to SVMs.

Table II summarizes the performance comparison (accuracy, sensitivity, and specificity) obtained with the semiautomated benchmark system, as well as with the fullyautomated SVM- and RVM-based systems. As can be seen, the RVM based system outperforms the SVM-based one across all three performance metrics. Relative to the benchmark system, the proposed RVM system achieved equal accuracy and improved sensitivity, with the advantage of not requiring human intervention. The benchmark system only slightly outperformed the fully-automated tools in terms of specificity, achieving 3\% higher performance relative to RVM. 


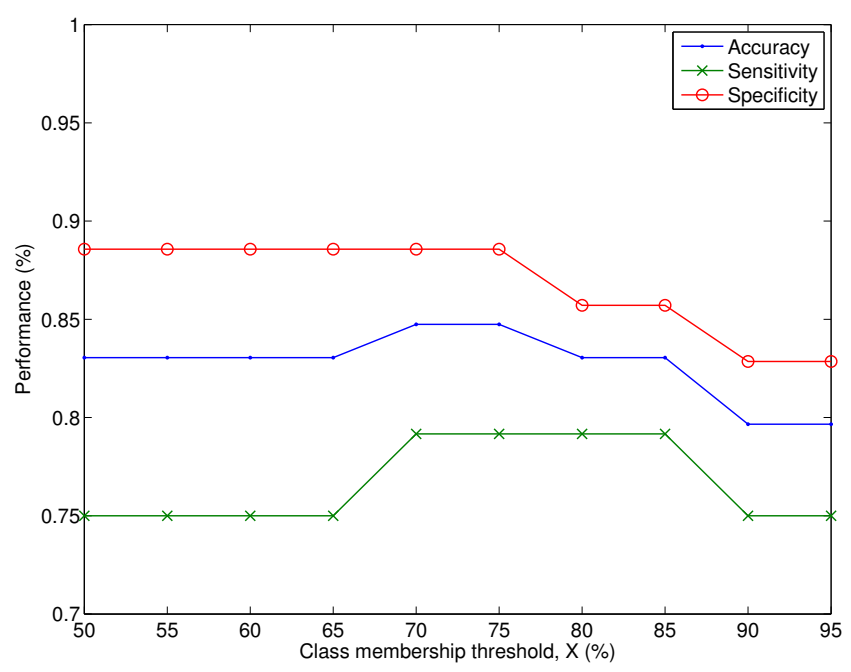

Fig. 4. RVM performance as a function of class membership threshold $X$.

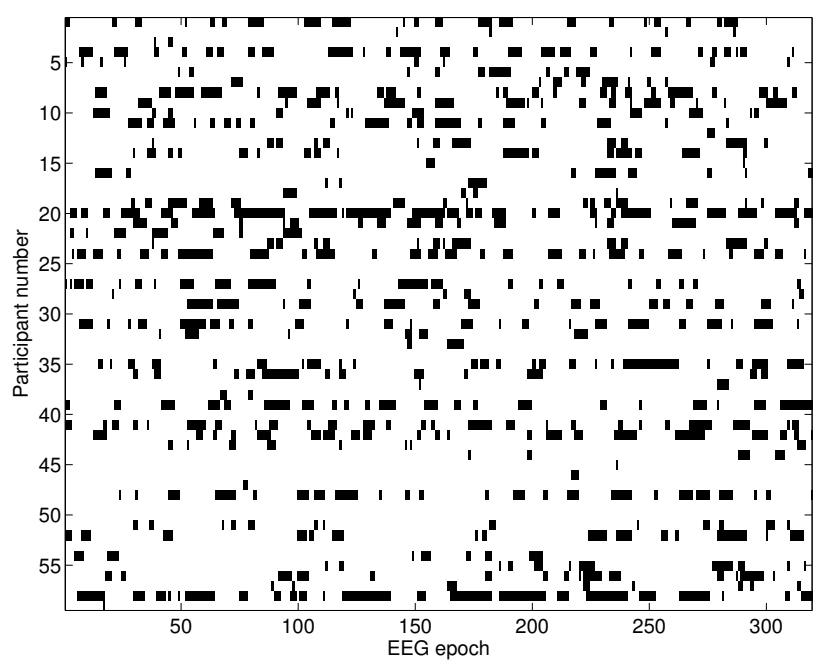

Fig. 5. Distribution plot of per-participant epochs classified as having more (unshaded) or less (shaded) than $75 \%$ class membership.

A closer look at the selected features from Table I shows that three features show up in both the manually-selected as well as wICA-processed top feature pool, namely: "O1_O2_theta_pwr", "PZ_alpha_pwr", and "F3 F4 delta cohe mag", thus showing the robustness of the bipolar $\mathrm{O} 1-\mathrm{O} 2$ and $\mathrm{F} 3-\mathrm{F} 4$ signals, along with the PZ alpha subband to EEG artefacts. Table III shows the distribution of the selected features across the different feature classes, brain regions, frequency subbands, and electrode montages.

TABLE II. PERFORMANCE COMPARISON OF SEMI-AUTOMATED BENCHMARK AND FULLY-AUTOMATED AD DIAGNOSTIC SYSTEMS.

\begin{tabular}{cccc}
\hline System & Accuracy (\%) & Sensitivity (\%) & Specificity (\%) \\
\hline \hline Benchmark & 84.7 & 75.0 & 91.4 \\
SVM & 81.4 & 75.0 & 85.7 \\
RVM & 84.7 & 79.2 & 88.6 \\
\hline
\end{tabular}

TABLE III. DISTRIBUTION OF SELECTED FEATURES USED WITH THE MANUALLY-SELECTED AND FULLY-AUTOMATED SYSTEMS DIVIDED BY FEATURE SET, BRAIN REGION, FREQUENCY BAND, AND MONTAGE.

\begin{tabular}{|c|c|c|}
\hline & \multicolumn{2}{|c|}{ Systems } \\
\hline & Manual & wICA-AAR \\
\hline \multicolumn{3}{|c|}{ NUMBER OF FEATURES PER FEATURE SET } \\
\hline spectral power & 10 & 14 \\
\hline coherence & 3 & 4 \\
\hline modulation & 11 & 6 \\
\hline \multicolumn{3}{|c|}{ NUMBER OF FEATURES PER BRAIN REGION } \\
\hline frontal & 8 & 9 \\
\hline central & 4 & 3 \\
\hline temporal & 3 & 6 \\
\hline parietal & 3 & 4 \\
\hline occipital & 6 & 2 \\
\hline \multicolumn{3}{|c|}{ NUMBER OF FEATURES PER FREQUENCY BAND } \\
\hline delta & 6 & 7 \\
\hline theta & 5 & 4 \\
\hline alpha & 3 & 4 \\
\hline beta & 10 & 9 \\
\hline \multicolumn{3}{|c|}{ NUMBER OF FEATURES FROM VIRTUAL CHANNELS } \\
\hline interhemispheric & 5 & 6 \\
\hline
\end{tabular}

From the manually-selected epochs, the amplitude modulation features are selected most often, followed by the spectral power based features. With the automated system, on the other hand, the spectral power features dominate, thus suggesting that amplitude modulation features may be sensitive to wICA artefact removal. Relative to brain regions, similar results are obtained with the two systems, with the exception of the temporal and occipital regions. For the manually-selected epochs, 3 of the 24 top features belonged to the temporal regions, whereas 6 belonged to the occipital region. With the wICA-processed epochs, on the other hand, this distribution was reversed with 6 of the top features belonging to the temporal region and only 2 to the occipital region. Regarding frequency bands and electrode montage (original versus virtual bipolar), the two systems obtained similar distributions and no effect could be observed from the automated artefact removal procedure. Combined, these findings suggest that a fully automated $\mathrm{AD}$ diagnostic system can be implemented with accuracies inline with those obtained with a semi-automated system relying on human intervention.

\section{COnClusion}

This paper has proposed a fully-automated EEG-based $\mathrm{AD}$ diagnosis system based on automatic artefact removal, automated feature selection, and relevance vector machine (RVM) based classification. Experimental results show the proposed system outperforming a benchmark algorithm based on support vector machines (SVM) and manually-selected artefact-free EEG epochs in terms of diagnostic accuracy and sensitivity. The proposed automated RVM-based system is also shown to outperform an automated SVM-based variant with the advantage of providing class membership probabilities. By providing such richer pool of information to clinicians, more accurate and earlier Alzheimer's disease assessments may be enabled, as well as disease progression monitoring. 


\section{ACKNOWLEDGMENT}

The authors acknowledge funding from the Natural Sciences and Engineering Research Council of Canada (NSERC), INRS-EMT, and the Foundation for Research Support of the State of São Paulo (FAPESP), Brazil.

\section{REFERENCES}

[1] Alzheimer's Association, "2013 Alzheimer's disease facts and figures," Journal of the Alzheimer's Association, vol. 9, no. 2, pp. 208-245, 2013.

[2] M. Hurd, P. Martorell, A. Delavande, K. J. Mullen, and K. M. Langa, "Monetary costs of dementia in the United States," New England Journal of Medicine, vol. 368, no. 14, pp. 1326-1334, 2013.

[3] WHO and Alzheimer's Disease International, "Dementia: a public health priority," World Health Organization, Tech. Rep., 2012.

[4] M. Sarazin, L. de Souza, S. Lehéricy, and B. Dubois, "Clinical and research diagnostic criteria for Alzheimer's disease," Neuroimaging clinics of North America, vol. 22, no. 1, pp. 23-32, 2012.

[5] T. H. Falk, F. J. Fraga, L. Trambaiolli, and R. Anghinah, "EEG amplitude modulation analysis for semi-automated diagnosis of Alzheimer's disease," EURASIP Journal on Advances in Signal Processing, vol. 2012, no. 1, pp. 1-9, 2012.

[6] C. Babiloni, R. Lizio, F. Vecchio, G. B. Frisoni, M. Pievani, C. Geroldi, F. Claudia, R. Ferri, B. Lanuzza, and P. M. Rossini, "Reactivity of cortical alpha rhythms to eye opening in mild cognitive impairment and Alzheimer's disease: an EEG study," Journal of Alzheimer's Disease, vol. 22, no. 4, pp. 1047-1064, 2010.

[7] J. Jeong, "EEG dynamics in patients with Alzheimer's disease," Clinical neurophysiology, vol. 115, no. 7, pp. 1490-1505, 2004.

[8] R. Brenner, R. Ulrich, D. Spiker, R. Sclabassi, C. Reynolds, R. Marin, and F. Boller, "Computerized EEG spectral analysis in elderly normal, demented and depressed subjects," Electroencephalography and clinical neurophysiology, vol. 64, no. 6, pp. 483-492, 1986.

[9] L. Coben, W. Danziger, and L. Berg, "Frequency analysis of the resting awake EEG in mild senile dementia of Alzheimer type," Electroencephalography and clinical neurophysiology, vol. 55, no. 4, pp. 372$380,1983$.

[10] S. Giaquinto and G. Nolfe, "The EEG in the normal elderly: a contribution to the interpretation of aging and dementia," Electroencephalography and clinical neurophysiology, vol. 63, no. 6, pp. 540546, 1986.

[11] C. Besthorn, H. Förstl, C. Geiger-Kabisch, H. Sattel, T. Gasser, and U. Schreiter-Gasser, "EEG coherence in Alzheimer's disease," Electroencephalography and clinical neurophysiology, vol. 90, no. 3, pp. 242-245, 1994.

[12] T. Locatelli, M. Cursi, D. Liberati, M. Franceschi, and G. Comi, "EEG coherence in Alzheimer's disease," Electroencephalography and clinical neurophysiology, vol. 106, no. 3, pp. 229-237, 1998.

[13] E. P. Sloan, G. W. Fenton, N. S. Kennedy, and J. M. MacLennan, "Neurophysiology and SPECT cerebral blood flow patterns in dementia," Electroencephalography and clinical neurophysiology, vol. 91, no. 3, pp. 163-170, 1994

[14] F. J. Fraga, T. H. Falk, L. R. Trambaiolli, E. F. Oliveira, W. H. Pinaya, P. A. Kanda, and R. Anghinah, "Towards an EEG-based biomarker for Alzheimer's disease: Improving amplitude modulation analysis features," in Proc. IEEE International Conference on Acoustics, Speech and Signal Processing. IEEE, 2013, pp. 1207-1211.

[15] J. Dauwels, K. Srinivasan, M. Ramasubba Reddy, T. Musha, F.-B Vialatte, C. Latchoumane, J. Jeong, and A. Cichocki, "Slowing and loss of complexity in Alzheimer's EEG: two sides of the same coin?" International journal of Alzheimer's disease, vol. 2011, 2011.

[16] H. Adeli, S. Ghosh-Dastidar, and N. Dadmehr, "Alzheimer's disease: models of computation and analysis of EEGs," Clinical EEG and Neuroscience, vol. 36, no. 3, pp. 131-140, 2005.

[17] I. Daly, N. Nicolaou, S. J. Nasuto, and K. Warwick, "Automated artifact removal from the electroencephalogram: A comparative study," Clinical EEG and Neuroscience, pp. 1-16, May 2013
[18] V. Krishnaveni, S. Jayaraman, L. Anitha, and K. Ramadoss, "Removal of ocular artifacts from EEG using adaptive thresholding of wavelet coefficients," Journal of Neural Engineering, vol. 3, no. 4, p. 338, 2006.

[19] G. Gómez-Herrero, W. de Clercq, H. Anwar, O. Kara, K. Egiazarian, S. Van Huffel, and W. Van Paesschen, "Automatic removal of ocular artifacts in the EEG without an EOG reference channel," in Proc. Nordic Signal Processing Symposium, 2006, pp. 130-133.

[20] W. de Clercq, A. Vergult, B. Vanrumste, W. Van Paesschen, and S. Van Huffel, "Canonical correlation analysis applied to remove muscle artifacts from the electroencephalogram," IEEE Transactions on Biomedical Engineering, vol. 53, no. 12, pp. 2583-2587, 2006.

[21] L. R. Trambaiolli, A. C. Lorena, F. J. Fraga, P. A. Kanda, R. Anghinah, and R. Nitrini, "Improving Alzheimer's disease diagnosis with machine learning techniques," Clinical EEG and Neuroscience, vol. 42, no. 3, pp. 160-165, 2011.

[22] C. Lehmann, T. Koenig, V. Jelic, L. Prichep, R. E. John, L.-O. Wahlund, Y. Dodge, and T. Dierks, "Application and comparison of classification algorithms for recognition of Alzheimer's disease in electrical brain activity (EEG)," Journal of neuroscience methods, vol. 161, no. 2, pp. 342-350, 2007.

[23] P. Kanda, L. Trambaiolli, A. Lorena, F. Fraga, L. Basile, R. Nitrini, and R. Anghinah, "Clinician's road map to wavelet EEG as an Alzheimer's disease biomarker," Clinical EEG and Neuroscience, 2013. [Online]. Available: http://eeg.sagepub.com/content/early/2013/10/15/1550059413486272

[24] G. McKhann, D. Drachman, M. Folstein, R. Katzman, D. Price, and E. M. Stadlan, "Clinical diagnosis of Alzheimer's disease report of the NINCDS-ADRDA work group under the auspices of department of health and human services task force on Alzheimer's disease," Neurology, vol. 34, no. 7, pp. 939-939, 1984.

[25] S. Brucki, R. Nitrini, P. Caramelli, P. Bertolucci, and I. Okamoto, "Suggestions for utilization of the mini-mental state examination in Brazil," Arquivos de neuro-psiquiatria, vol. 61, pp. 777-781, 2003.

[26] P. L. Nunez, Electric fields of the brain: the neurophysics of EEG. Oxford University Press, 2006.

[27] R. Cassani, T. H. Falk, F. J. Fraga, P. A. Kanda, and R. Anghinah, "The effects of automated artifact removal algorithms on electroencephalography-based Alzheimer's disease diagnosis," Frontiers in aging neuroscience, under review.

[28] N. Castellanos and V. Makarov, "Recovering EEG brain signals: artifact suppression with wavelet enhanced independent component analysis," Journal of neuroscience methods, vol. 158, no. 2, pp. 300-312, 2006.

[29] V. A. Makarov, "Wavelet enhanced independent component analysis (wICA) package for matlab," 2012.

[30] L. Sörnmo and P. Laguna, Bioelectrical signal processing in cardiac and neurological applications. Academic Press, 2005.

[31] J. Dauwels, F. Vialatte, T. Musha, and A. Cichocki, "A comparative study of synchrony measures for the early diagnosis of Alzheimer's disease based on EEG," Neurolmage, vol. 49, no. 1, pp. 668-693, 2010.

[32] F. J. Fraga, T. H. Falk, P. A. M. Kanda, and R. Anghinah, "Characterizing Alzheimer's disease severity via resting-awake EEG amplitude modulation analysis," PLOS ONE, vol. 8, p. e72240, 2013.

[33] L. R. Trambaiolli, T. H. Falk, F. J. Fraga, R. Anghinah, and A. C. Lorena, "EEG spectro-temporal modulation energy: A new feature for automated diagnosis of Alzheimer's disease," in Proc International Conference of the IEEE Engineering in Medicine and Biology Society. IEEE, 2011, pp. 3828-3831.

[34] E. Bedrosian, "A product theorem for Hilbert transforms," Proceedings of the IEEE, vol. 51, no. 5, pp. 868-869, 1963.

[35] C. Cortes and V. Vapnik, "Support-vector networks," Machine learning, vol. 20, no. 3, pp. 273-297, 1995.

[36] M. Tipping, "Sparse Bayesian learning and the relevance vector machine," Journal Machine Learning Research, vol. 1, pp. 211-244, 2001. 